\title{
Fertility preservation in Hodgkin's lymphoma patients that undergo targeted molecular therapies: an important step forward from the chemotherapy era
}

This article was published in the following Dove Press journal:

Cancer Management and Research

\begin{abstract}
Alexandra Traila ${ }^{1,2, *}$
Delia Dima ${ }^{3}$

Patriciu Achimas-Cadariu ${ }^{1,2, *}$

Romeo Micul,4

'School of Medicine, luliu Hatieganu University of Medicine and Pharmacy, Cluj Napoca, Romania; ${ }^{2}$ Department of Surgical Oncology, Ion Chiricuta Oncology Institute, Cluj Napoca, Romania; ${ }^{3}$ Department of Hematology, Ion Chiricuta Oncology Institute, Cluj Napoca, Romania; ${ }^{4}$ Department of Human Assisted Reproduction of Ist Gynecology Clinic, Cluj Napoca, Romania

*These authors contributed equally to this work
\end{abstract}

Correspondence: Delia Dima Department of Hematology, Ion Chiricuta Oncology Institute, Bulevardul 21 Decemberi $\mathrm{Nr} 73$, Cluj Napoca, Romania

Tel +40 722957677

Email delia.monica.dima@gmail.com

\begin{abstract}
In total, $80 \%-90 \%$ of Hodgkin's lymphoma (HL) patients are curable with combination chemoradiotherapy. Due to improvements in therapeutic strategies, $50 \%$ of all relapsed/ refractory patients may undergo complete clinical responses and have long-term survival. Treatment options for HL are effective, but may have a negative impact on post-chemotherapy fertility. Thus, cryopreservation of semen prior to treatment is recommended for male patients. For female patients, assisted reproductive techniques (ART) consult and fertility preservation should be offered as a therapeutical option. In the last years, new targeted molecules have been available for HL treatment. These new drugs showed a high rate of overall responses in the setting of heavily pretreated patients, most of them in relapse after autologous stem cell transplantation, a group previously considered very poor risk. Up to $50 \%$ of patients have a complete response and an improved overall survival. Future studies will address the usefulness of novel molecules as a frontline therapy. Considering the high response and survival rates with monoclonal antibody-based therapeutics, fertility has become a concerning issue for long-term HL survivors. As progress has been made regarding ART, with the rigorous steps planned for HL patients, more survivors will become parents.
\end{abstract}

Keywords: Hodgkin's lymphoma, infertility, pregnancy, fertility preservation

\section{Introduction}

Due to modern combined chemoradiotherapy strategies, Hodgkin's lymphoma (HL) is now considered to be a malignant disease with a high curability rate and a 5-year progression free survival of $87 \% .{ }^{1}$ Patients diagnosed with early stage HL are generally treated with short courses of chemotherapy plus consolidation radiotherapy. Patients with an advanced stage disease are treated with combination chemotherapy. ${ }^{2-4}$ The progress made in the last few years with intensive chemotherapy, autologous stem cell transplantation (ASCT), and novel targeted molecules has improved the response and survival rates, even for advanced stage of relapsed/refractory HL. ${ }^{5-9}$ Patients with progressive disease after salvage therapy were considered, a decade ago, to be a very poor prognosis group. Eligible patients are now being offered the chance of undergoing an allogeneic stem cell transplantation, in association with an increased risk of therapy related-mortality. ${ }^{10-12}$ Targeted molecules show unprecedented response rates in present-day chemotherapy. ${ }^{13-17}$ Still, large cohort studies have yet to address the concerning issue of long-term complications. One such complication is infertility in both men and women. In women, infertility may 
be due to chemotherapy-induced diminished ovarian reserve (chDOR) or to premature ovarian failure (POF). chDOR manifests as secondary amenorrhea and persisting high levels of gonadotropins. ${ }^{18}$ POF is defined as the loss of ovarian function of peripheral origin before the age of 40 . In men, chemotherapy may be complicated by temporary or definitive azoospermia. Even more, there is the issue of subfertility, a known complication of HL that affects both sexes, predominantly men. A possible explanation would be the disturbed cellular immunity. ${ }^{19-21}$

In the current paper, we aim to review the current knowhow on fertility complications induced by chemotherapy and radiotherapy, as well as by novel drugs approved for HL therapy. Other possible treatment options, such as assisted reproductive techniques (ART) for HL patients with fertility problems and long-term survivors, are discussed, with the purpose of designing an algorithm with distinct steps on HL diagnosis, treatment, and tissue/sperm preservation.

\section{Methods}

The analysis was made following an extensive search of the National Library of Medicine's MEDLINE database using PubMed and Google Scholar, as previously described. ${ }^{22}$ Papers included in the analysis were limited to English, German, Romanian, and French language publications, but were not limited to any geographical region, from January 1976 to July 2017. Only papers published between 1976 and 2017 were considered in order to avoid any inconsistencies in diagnostic criteria and also cover the period of publications on HL. The search strategy was based on the combination of the keywords "Hodgkin's lymphoma", "infertility", "pregnancy", "fertility preservation". Subsequently, an additional manual search of the citations of the previously selected papers was performed.

We identified 268 candidate papers, of which nine papers were excluded as they were related to pediatric oncology. Nine other papers related to other types of lymphoma and solid cancers, being excluded, raised the number of analyzed papers to 250 .

\section{Current knowledge on fertility and HL chemotherapy}

The risk of infertility depends on patient's age, type, and dose of chemotherapy. ${ }^{23-28}$ The risk of infertility is higher after the age of 30, as the follicle reserve diminishes with age. ${ }^{29-33} \mathrm{~A}$ large Norwegian study enrolled women diagnosed with and treated for HL, and reported that patients aged under 25 years had the same risk of infertility as the patients older than 30 years, but with delayed complications (15 vs 2 years). ${ }^{34}$

In men, spermatogonia is constant throughout life. ${ }^{35}$ In a recent study by the European Organization for Research and Treatment of Cancer Lymphoma Group (EORTC) and Groupe d'Etude des Lymphomes de l'Adultes (GELA), risk factors associated with infertility in men treated for HL were considered to be the general symptoms at diagnosis, especially fever and night sweats, and an increased erythrocyte sedimentation rate (ESR), probably due to proinflammatory cytokines. ${ }^{36}$

Early stage HL is treated with the ABVD (doxorubicine, bleomycin, vinblastine, dacarbazine) protocol as the standardof-care. ${ }^{37,38}$ Hodgson et al, ${ }^{39}$ as well as Brusamolino et al, ${ }^{40}$ have shown that ABVD chemotherapy is safe and birth rates are comparable with the general population. Escalated BEACOPP (bleomycine, etoposide, doxorubicine, cyclophosphamide, vincristine, procarbazine, prednisone) protocol is used for advanced stage HL, which, according to a multivariate analysis of a German cohort, is positively associated with the development of consequent infertility. ${ }^{41}$ Similar results were later reported by Decanter et al, ${ }^{42}$ a group that correlated a decrease in the serum levels of anti-Müllerian hormone $(\mathrm{AMH})$ in patients treated with both ABVD and alkylating agents-containing regimens, but the recovery was complete after 1 year in the ABVD group. ${ }^{42}$ The alkylating agent cyclophosphamide is known to have a high risk of gonadotoxicity. ${ }^{43}$ Cyclophosphamide alters the ovarian reserve in a dose-, duration-, and age-dependent manner; $40 \%$ of female patients under the age of 40 years have developed chDOR after cyclophosphamide-based chemotherapy regimens. Alkylating agents induce a risk of infertility of 3.98 when compared to the general population, with the risk increasing due to the cumulative dose. ${ }^{44,45}$

For men treated with the ABVD protocol, the recovery of spermatogenesis is similar with the recovery of ovarian function in women, 6-18 months following the end of therapy. van der Kaaij et $\mathrm{al}^{36}$ report a recovery time for the folliclestimulating hormone (FSH) of 18 months for $82 \%$ of male patients treated without alkylating agents vs 27 months for $30 \%$ of patients treated with alkylating agent-containing regimens. ${ }^{36}$ As in female patients, alkylating agents and platinum-based regimens are the most important risk factor for infertility in men, with the risk being dose-dependent. The decline in sperm count was reported after 2-3 months of chemotherapy. The corticosteroids included in the escalated BEACOPP regimen also have an inhibitor effect on the hypothalamic-pituitary-gonadal axis of male patients. ${ }^{46,47}$ 
An additional risk factor for therapy-related infertility is pelvic radiotherapy. For male patients, recent progress in ART could lead to an improved rate of fatherhood following treatment, even in oligozoospermic patients. Radiotherapy is used in combination with chemotherapy for early stage $\mathrm{HL}$, as well as for bulky metastatic lymph nodes in advanced stage HL. A dose of $2.5-5$ Gy is associated with infertility in $30 \%-40 \%$ of women aged $15-40$ years and in $90 \%$ of women older than 40 years. Radiation is toxic, both for active and dormant follicles. For men, irradiation with 1-2 Gy is associated with a consequent risk of sterility. ${ }^{48}$ A 7.5 Gy radiation to the testis induces the highest risk of sterility. ${ }^{47}$ The data regarding the stage of the disease is controversial. Some studies report no association between disease stage and fertility, but probably the risk associated with disease stage is related to the therapy used.

Second-line therapies include DHAP (dexamethasone, high-dose cytarabine, cisplatin), ICE (ifosfamide, carboplatin, etoposide), IGEV (ifosfamide, gemcitabine, etoposide, vinorelbine), GDP (gemcitabine, dexamethasone, cisplatin), GVD (gemcitabine, vinorelbine, dexamethasone), MINE (mesna, ifosfamide, novantrone, etoposide) chemotherapy regimens, none of which have yet been evaluated for the risk of infertility. The infertility risk associated with an ASCT was not prospectively evaluated so far, but few case reports of pregnancies after BEAM (BCNU, etoposide, cytarabine, melphalan) conditioning chemotherapy have been published. ${ }^{49,50}$ Several studies and reports of the European Blood and Marrow Transplantation (EBMT) have shown a high risk of infertility associated with allogeneic transplantation, especially when total body irradiation (TBI) was used as part of the conditioning regimen. ${ }^{49}$ However, up to $20 \%-25 \%$ of patients recover their fertility several years after myeloablative allogeneic transplantation. ${ }^{47}$

Overall, on a Dutch cohort, de Bruin et $\mathrm{al}^{51}$ have shown that 97 out of 518 patients treated with chemotherapy for HL developed a premature menopause. The largest study that evaluated the risk of infertility in patients treated for $\mathrm{HL}$ was published by Swerdlow et al. ${ }^{50}$ The study evaluated 2,127 female patients treated from 1960 to 2004, with the evaluation being made from 2003 to 2012. Among these patients, 1,292 developed early or premature menopause. The group showed a cumulative risk of infertility associated with age, at least six cycles of alkylating agent therapy, BEAM chemotherapy, or 5 Gy pelvic radiotherapy. There was an increased risk for older patients, but there was also evidence of cumulative incidence years after treatment.

\section{Targeted new therapies and fertility}

In the last 2 years, important progress has been made in the treatment of HL with monoclonal antibody-based drugs. These novel targeted molecules have shown unprecedented overall response rates for heavily pretreated patients, most of whom are in relapse after multiple lines of chemotherapy and ASCT. $^{52,53}$ It is too early to have fertility studies considering the limited experience, but specialists and patients should be aware of safety information regarding pregnancies. Some information is available for rituximab, in use since 1997 for different Non-Hodgkin's lymphoma (NHL) subtypes, and rituximab appears to be safe. ${ }^{54,55}$ There is limited data on fertility issues, but the available animal studies show embryofetal abnormalities correlated to targeted therapy. The anti PD-1 molecules are IgG4 that crossed the placental barrier, and monomethyl auristatin E (MMAE) has proven testicular toxicity.

Brentuximab vedotin is an anti-CD30 antibody drug conjugate, covalently linked to a antimicrotubule agent monomethyl auristatin $\mathrm{E}$ (MMAE), with proven efficacy in CD30 lymphoproliferative diseases such as HL, anaplastic large cell lymphoma (ALCL), and other types of non-Hodgkin's lymphoma (NHL). ${ }^{56-59}$ This drug is approved for relapsed/ refractory HL after ASCT or after two prior lines of chemotherapy, brentuximab vedotin showed an overall response rate of $75 \%$ and a complete response rate of $34 \%$, in phase 2 trials as a single agent. ${ }^{60,61}$ Recent results of the AETHERA study group suggest a role of brentuximab vedotin treatment as consolidation after ASCT for high risk patients. ${ }^{62} \mathrm{CD} 30$ is not expressed in physiological conditions, with some exceptions such as decidual cells in the uterus and endometrium during pregnancy. ${ }^{63,64}$

Up to this point, no studies have been published regarding the use of brentuximab in pregnant women, but preclinical studies on animal models have shown significantly decreased embryo viability and fetal malformations. ${ }^{61}$ Thus, both HL and ALCL patients are advised not to become pregnant during brentuximab therapy and 6 months after the last dose. For men treated with brentuximab, the same rules as for chemotherapy should be applied regarding sperm collection, since non-clinical studies have revealed testicular toxicity. MMAE has aneugenic properties leading to testicular atrophy and degeneration, that are partially reversible. Male patients should use contraception methods for at least 6 months after the last dose. There is no information on breastfeeding, but it is possible that a very low quantity of brentuximab could be found in milk, since it is a large protein. 
Brentuximb is an important acquisition for the HL treatment, but, regarding conception, the same rules as for chemotherapy should be applied.

Nivolumab is a fully human anti PD-1-monoclonal antibody, used in clinical trials for the treatment of HL in the setting of relapsed/refractory disease after ASCT followed by brentuximab vedotin therapy. ${ }^{65} \mathrm{In}$ a recent large Phase II clinical trial, 80 patients who failed both ASCT and brentuximab vedotin have received nivolumab. The overall response rate was $66 \%$, with a complete response of $8.8 \%$ and partial response of $57.5 \%$ of patients. The 6-month overall survival was $99 \% .{ }^{66-68}$ So far, there are no studies on fertility in men or women treated with nivolumab. There is no data on pregnancy outcome under nivolumab treatment, but animal studies show embryofetal toxicity. ${ }^{69}$ The only published literature is from various regulatory agencies recommendations for contraception during nivolumab therapy and 5 months after the last dose.

Pembrolizumab is another anti PD-1 monoclonal antibody tested in patients with HL. ${ }^{70}$ Recently, the results from the Keynote-013 Phase I/II clinical trial have been reported with impressive outcome. ${ }^{71}$ Among the 31 patients treated with pembrolizumab, all of them after relapse from ASCT, the overall response rate was $65 \%$, with $16 \%$ complete response and $48 \%$ partial response rates. ${ }^{72}$ Still, there is no available clinical data on fertility under pembrolizumab therapy, but animal studies have revealed no negative effects. As with nivolumab, there are no data on the use of pembrolizumab in pregnant women, but animal studies reveal fetal harm and fetal loss. So far, recommendations treatment with pembrolizumab is not to be used in pregnancies.

Still, both anti PD-1 antibodies are IgG4, known to cross the placental barrier. ${ }^{73,74}$ PD-1 blockade with nivolumab or pembrolizumab is safe and effective. Treatment is usually administered for 3-6 months, up to 2 years. Retreatment is also allowed in the case of initial response, with most frequent adverse events being the immune reactions. It is important to emphasize that patients treated with anti PD-1 monoclonal antibodies are heavily pretreated with chemotherapy and ASCT, both therapies known to impair fertility in men and women. Current studies evaluate the role of anti PD-1 monoclonal antibodies as first-line therapy. We hope for new data on fertility complications due to these targeted molecules.

Rituximab is a chimeric mouse/human IgG1k monoclonal antibody targeting the $\mathrm{B}$ cell surface antigen CD20. ${ }^{75-77}$ Rituximab is used in the clinic for treating diffuse large $\mathrm{B}$ cell NHL, follicular NHL, as well as for nodular lymphocyte predominant HL. The median half-life is $18-22$ days, but the drug can be detected in blood up to 24 weeks after administration. The $\mathrm{B}$ cell depletion induced by rituximab can last for 6 months to years in some patients. Chakravarty et $\mathrm{al}^{78}$ have shown that most of the 231 pregnancies included in the study, with preconceptional and antepartum exposure to rituximab, resulted in uncomplicated live births. In this study, there was no pattern of congenital abnormalities identified and associated with rituximab. There was also no pattern of neonatal infections, but cytopenias were detected in seven of the eleven reports. This observation has led to the recommendation of blood count for all newborns exposed to rituximab, especially shortly before or during gestation. Given the prolonged B-cell depletion after rituximab administration, regular check-ups for both the mother and the newborn should be performed. No clear conclusion can be drawn from current reports regarding male exposure to rituximab, and data are still insufficient for the evaluation of gonadal toxicity in men. ${ }^{78}$

\section{Gonadal function evaluation and fertility preservation options}

There is no consensus on ideal parameters of fertility. ${ }^{79} \mathrm{FSH}$ is elevated in the case of impaired ovarian function, and it has been used as the most important ovarian function parameter, but it's high intercycle variability makes this serum analysis unreliable. Currently, AMH is being used as the best tool for assessment of ovarian function, as it demonstrated high sensitivity and stability. ${ }^{80}$ Another evaluation assay is the determination of inhibin $\mathrm{B}$ hormone, secreted by the follicles recruited during the ovarian cycle and involved in the negative regulation of FSH. Nevertheless, this type of analysis is not available in all laboratories, especially in developing economies. ${ }^{81}$ The transvaginal ultrasound performed at day 3 of the menstrual cycle provides the number of follicles between 2 and $10 \mathrm{~cm}$, that correlate with the ovarian reserve. ${ }^{82}$ Unfortunately, in the case of amenorrhea, the ultrasound has not proven to be useful. Most specialized centers in reproductive techniques use both AMH and/or FSH serum levels and ovarian ultrasound. ${ }^{83,84}$

For male patients, the best available assessment is semen analysis, which provides data on sperm count, as well as vitality and mobility of the spermatozoids. The test should be performed at least 3 months after chemotherapy, as spermatogenesis takes $\sim 74$ days. FSH and inhibin B serum levels could also be used.

The most important step on fertility preservation for patients treated for $\mathrm{HL}$ is the multidisciplinary collaboration between hematologist, ART specialist, and gynecologist. The 
use of gonadotropin-releasing hormone analogs ( $\mathrm{GnRH}-\mathrm{a})$ during chemotherapy is still controversial. ${ }^{85,86}$ Several randomized trials suggest the efficacy of GnRH-a in the reduction of chDOR risk for female patients undergoing chemotherapy, based on a chemical-induced menopause which can protect the ovary from the cytotoxic effect. A German trial has evaluated the use of oral contraceptives in comparison to $\mathrm{GnRH}$-a for female patients treated with escalated BEACOPP, and found no efficacy of GnRH-a. Currently, there is no evidence that $\mathrm{GnRH}-\mathrm{a}$ administration during chemotherapy could increase the rate of pregnancies, with no clear recommendation regarding their use. Still, important advantages for the clinician are the good control of the menstrual cycles and the reduction of irregular bleeding. ${ }^{87-91}$

Currently, three preservation methods are available for female patients: oocyte cryopreservation, in vitro fertilization for embryo cryopreservation, and cryopreservation of ovarian tissue. Recently, a new technique of retrieval of cumulus oocyte complexes, followed by in vitro maturation and vitrification, in combination with ovarian tissue cryopreservation, was reported in France. ${ }^{92,93}$ With regard to oocyte cryopreservation, ovarian stimulation for 4-6 weeks is still needed, and the success rate is only $3 \% .{ }^{94,95}$ Fertilization of the oocyte by intra-cytoplasmic sperm injection and subsequent vitrification could increase the success rate to $6.8 \% .{ }^{96}$ The in vitro fertilization followed by embryo cryopreservation is a feasible option for patients with a stable partner, where a delay in chemotherapy is not contraindicated, and where an ovarian stimulation of 9-14 days, but sometimes 4-6 weeks, is possible. The success rate has been reported to be $18 \%{ }^{20}$

The cryopreservation of the ovarian tissue is a new promising procedure, but it's results need further confirmation, since only a few pregnancies have been reported so far, with a risk of $50 \%$ loss of ovarian reserve and an additional risk of malignant cell reimplantation..$^{97}$

For male patients that undergo chemotherapy, semen collection and preservation must be proposed to the patient prior to therapy. There is a known risk of subfertility in HL patients, ${ }^{19,20}$ and normal sperm is essential for embryo development. ${ }^{98,99}$ It is of utmost importance to perform quality control assays prior to preservation. There is no consensus on the ideal method of quality measurement and there are several available methods such as the SCSA (sperm chromatin structure assay; ${ }^{100}$ the detection of single and double DNA breaks by terminal deoxynucleotidyl transferasemediated dUTP nick end labeling (TUNEL) ${ }^{101}$ the comet assay, ${ }^{102}$ or flow cytometry using monobromobimane for reactive $\mathrm{SH}$ groups in protamines and using chromomycin A3 for DNA compaction. ${ }^{103} \mathrm{O}^{\prime}$ Flaherty et $\mathrm{al}^{19}$ have reported increased DNA damage in newly diagnosed HL patients when compared with controls, using two control methods: comet assay and flow cytometry with monobromobimane and chromomycin. Semen collection may be possible after initiation of chemotherapy, but the risk of genetic defects in the offspring is unknown. ${ }^{19}$ Some groups report success with cryopreservation and subsequent transplantation of spermatogonial stem cells, but these options are still experimental and are offered only to patients in whom semen cryopreservation is not possible. ${ }^{104}$ As in female patients, there is limited data regarding the efficacy of hormone suppression in reducing the risk of infertility during chemotherapy. ${ }^{105,106}$ All patients should be offered the possibility of sperm preservation, with the best local available quality control assay.

The American Society of Clinical Oncology published in 2013 updated recommendations on fertility issues in patients with cancer. For men, sperm cryopreservation is the recommended method as the only proven fertility preservation method. For women, embryo and oocyte cryopreservation are established fertility preservation methods. The authors suggest ovarian transposition in the case of pelvic radiotherapy. Nor for men or women, there is no recommendation for hormonal suppression, since there is insufficient data on effectiveness. $^{107}$

Figures 1 and 2 present the algorithms for fertility preservation in HL patients, as a proof-of-concept.

\section{Conclusion}

Treatment of young patients diagnosed with HL is multidisciplinary and involves a team of hematologists, gynecologists, and fertility specialists, who should all keep in mind that increased disease activity could be associated with adverse pregnancy outcome. ${ }^{108-113}$ The risk of chemotherapy-induced infertility should be discussed with all newly diagnosed HL patients. A thorough evaluation of ovarian function and semen should be performed in all young HL patients. Even if the infertility risk associated with ABVD regimen is known to be low, fertility issues and preservation methods should be discussed with all patients under the age of 40 diagnosed with early stage HL before the beginning of therapy. Patients diagnosed with advanced stage HL, treated with combination chemotherapy, should also be offered fertility preservation methods prior to therapy, as well as counseling, which must be offered to all patients regarding the risks of pregnancy during treatment. Other 


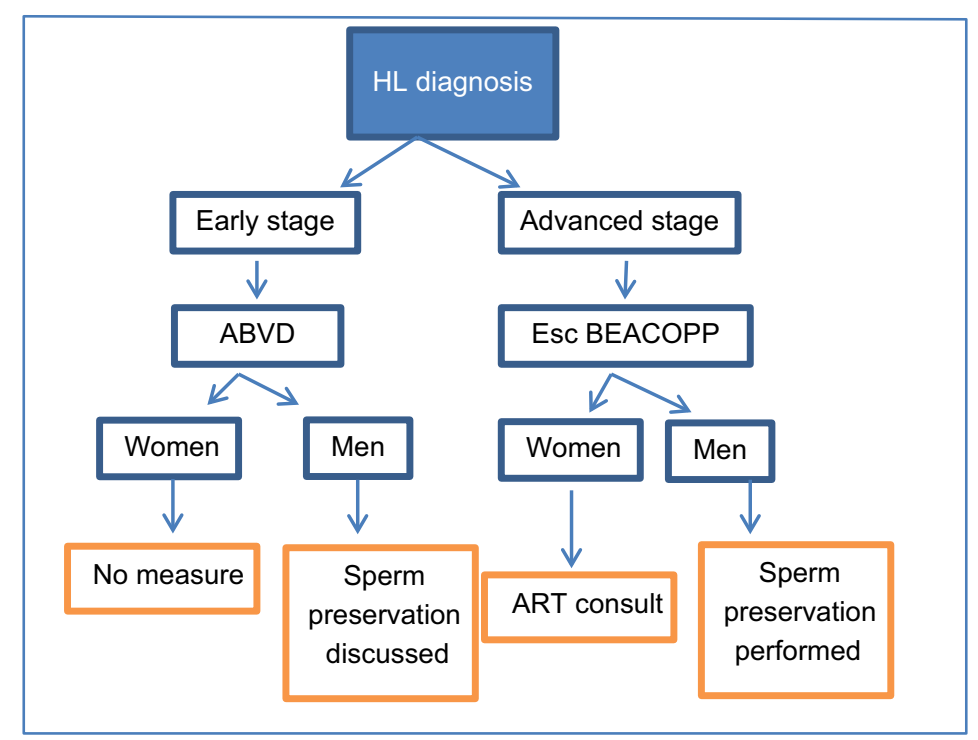

Figure I Algorithm for fertility preservation in $\mathrm{HL}$ patients, at diagnosis.

Abbreviations: HL, Hodgkin's lymphoma; ABVD, doxorubicine, bleomycin, vinblastine, dacarbazine; Esc BEACOPP, escalated bleomycine, etoposide, doxorubicine, cyclophosphamide, vincristine, procarbazine, prednisone; ART, assisted reproductive techniques.

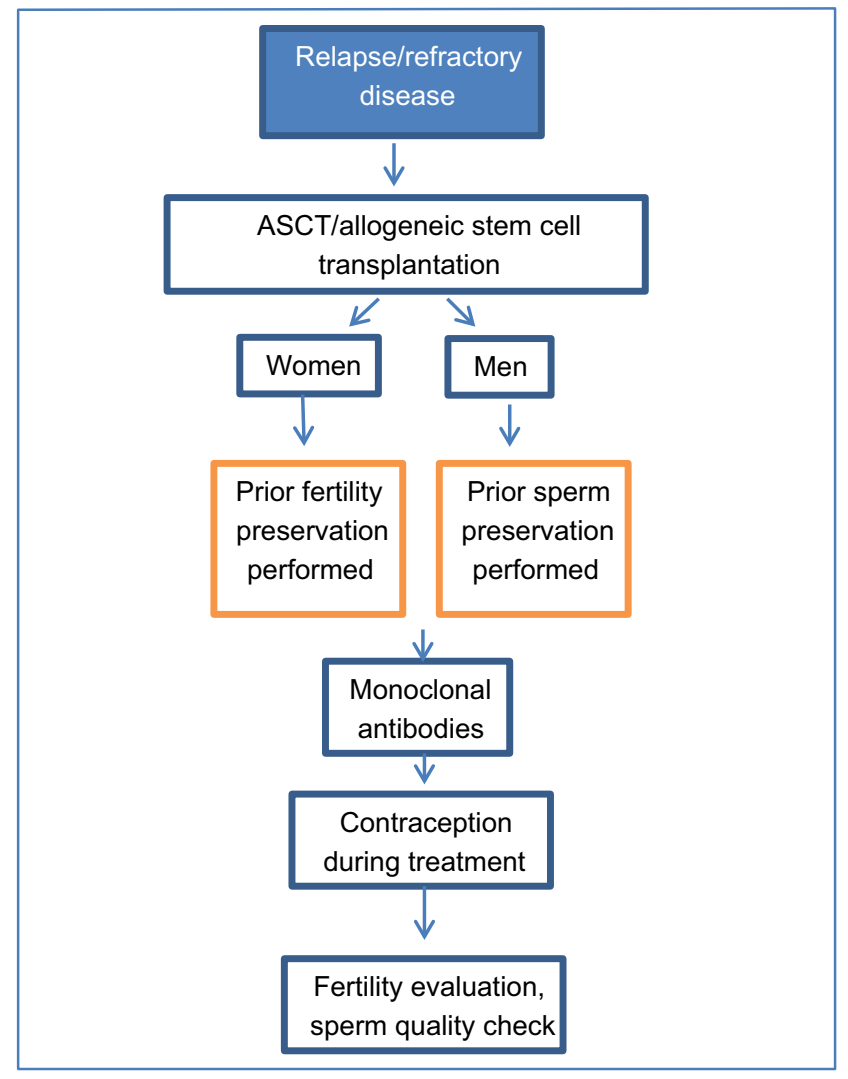

Figure 2 Algorithm for fertility preservation in relapsed/refractory HL patients. Abbreviations: HL, Hodgkin's lymphoma; ASCT, autologous stem cell transplantation.

long-term complications of chemoradiotherapy, such as secondary acute leukemia or breast cancer or thyroid disfunction, should be kept in mind when deciding in favor of fertility preservation at the initial HL diagnosis, consider- ing the timeframe for next chemotherapy and the possible contraindication for ovarian stimulation.

Current recommendations suggest a planned pregnancy after 6-24 months following chemotherapy, considering the approximate 6 months interval for follicullar maturation and the relapse risk which is highest during the first 2 years. For the anti-CD20 monoclonal antibody rituximab, the recommendations are for contraception during treatment and no less than 12 months after the last dose. For anti-PD-1 monoclonal antibodies, strong recommendations are in favor of contraception. Still, most available data on fertility issues and pregnancy rate and pregnancy outcome are from voluntary reports, from clinical trials, or from registries, thus making clear interpretation of data difficult. Long-term follow-ups of pregnancies during chemo-, immunotherapy are scarce, and reports on pregnancies and their outcomes should be encouraged, in order to have better guidelines.

With the impressive results obtained with targeted molecules, even in the setting of relapsed/refractory disease, longterm survivors of HL will be seen. Future studies will assess the best approach regarding the use of monoclonal antibodies in frontline or relapsed settings, with single agents or combination therapy. Due to the important progress made with the addition of monoclonal antibodies, fertility issues need to be carefully studied in future trials for responding patients.

\section{Acknowledgment}

All authors have read and approved the final version of the manuscript. 


\section{Disclosure}

The authors report no conflicts of interest in this work.

\section{References}

1. Engert A, Diehl V, Franklin J, et al. Escalated-dose BEACOPP in the treatment of patients with advanced-stage Hodgkin's lymphoma: 10 years of follow-up of the GHSG HD9 study. J Clin Oncol. 2009;27(27):4548-4554.

2. Donato EM, Fernandez-Zarzoso M, De La Rubia J. Immunotherapy for the treatment of Hodgkin lymphoma. Expert Rev Hematol. 2017;10(5):417-423.

3. Phillips T, Mercer J. Surveillance scans in lymphoma: friend or foe? Curr Treat Options Oncol. 2017;18(2):10.

4. Johnson P, Longley J. Should response-adapted therapy now be the standard of care for advanced Hodgkin's lymphoma? Curr Treat Options Oncol. 2017;18(3):15.

5. Brammer JE, Khouri I, Gaballa S, et al. Outcomes of haploidentical stem cell transplantation for lymphoma with melphalan-based conditioning. Biol Blood Marrow Transplant. 2016;22(3):493-498.

6. Fetica B, Achimas-Cadariu P, Pop B, et al. Non-Hodgkin lymphoma in Romania: a single-centre experience. Hematol Oncol. 2017;35(2):198-205.

7. Tomuleasa C, Akin-Abayomi E, Dima D, et al. Do lymphomas have an autoimmune background and how can we increase the sensitivity of the diagnosis of primary pancreatic lymphoma with relatively small samples? J Gastrointestin Liver Dis. 2015;24(3):391-392.

8. Grewal R, Cucuianu A, Swanepoel C, et al. The role of microRNAs in the pathogenesis of HIV-related lymphomas. Crit Rev Clin Lab Sci. 2015;52(5):232-241.

9. Tanase A, Tomuleasa C, Marculescu A, Bardas A, Colita A, Ciurea SO. First successful haploidentical stem cell transplantation in Romania. Rom J Intern Med. 2016;54(3):194-200.

10. Giaccone L, Festuccia M, Zallio F, et al. Long-term follow-up of allogeneic stem cell transplantation in relapsed/refractory Hodgkin lymphoma. Bone Marrow Transplant. 2017;52(8):1208-1211.

11. Gaballa S, Ge I, El Fakih R, et al. Results of a 2-arm, phase 2 clinical trial using post-transplantation cyclophosphamide for the prevention of graft-versus-host disease in haploidentical donor and mismatched unrelated donor hematopoietic stem cell transplantation. Cancer. 2016;122(21):3316-3326.

12. Tanase A, Tomuleasa C, Marculescu A, et al. Haploidentical donors: can faster transplantation be life-saving for patients with advanced disease? Acta Haematol. 2016;135(4):211-216.

13. Aldea $\mathrm{M}$, Craciun $\mathrm{L}$, Tomuleasa $\mathrm{C}$, et al. Repositioning metformin in cancer: genetics, drug targets, and new ways of delivery. Tumour Biol. 2014;35(6):5101-5110.

14. Gafencu GA, Tomuleasa CI, Ghiaur G. PARP inhibitors in acute myeloid leukaemia therapy: How a synthetic lethality approach can be a valid therapeutic alternative. Med Hypotheses. 2017;104: $30-34$.

15. Nagy-Simon T, Tatar AS, Craciun AM, et al. Antibody conjugated, raman tagged hollow gold-silver nanospheres for specific targeting and multimodal dark-field/SERS/two photon-FLIM imaging of CD19(+) B lymphoblasts. ACS Appl Mater Interfaces. 2017;9(25): 21155-21168.

16. Petrushev B, Boca S, Simon T, et al. Gold nanoparticles enhance the effect of tyrosine kinase inhibitors in acute myeloid leukemia therapy. Int J Nanomedicine. 2016;11:641-660.

17. Aldea MD, Petrushev B, Soritau O, et al. Metformin plus sorafenib highly impacts temozolomide resistant glioblastoma stem-like cells. J BUON. 2014;19(2):502-511.

18. Lutchman Singh K, Davies M, Chatterjee R. Fertility in female cancer survivors: pathophysiology, preservation and the role of ovarian reserve testing. Hum Reprod Update. 2005;11(1):69-89.
19. O'Flaherty C, Vaisheva F, Hales BF, Chan P, Robaire B. Characterization of sperm chromatin quality in testicular cancer and Hodgkin's lymphoma patients prior to chemotherapy. Hum Reprod. 2008;23(5):1044-1052.

20. Fabbri R, Pasquinelli G, Magnani V, et al. Follicle features in adolescent and young adult women with Hodgkin's disease prior to chemotherapy: a preliminary report. Reprod Biomed Online. 2011;23(6):799-805.

21. Peccatori FA, Azim HA Jr, Orecchia R, et al. Cancer, pregnancy and fertility: ESMO Clinical Practice Guidelines for diagnosis, treatment and follow-up. Ann Oncol. 2013;24(Suppl 6):vi160-170.

22. Dima D, Tomuleasa C, Frinc I, et al. The use of rotation to fentanyl in cancer-related pain. J Pain Res. 2017;10:341-348.

23. Huser M, Smardova L, Ventruba P, Mayer J. [Impact of oncological treatment on human reproduction]. Klin Onkol. 2010;23(3):165-170.

24. Huser M, Zakova J, Smardova L, et al. Combination of fertility preservation strategies in young women with recently diagnosed cancer. Eur J Gynaecol Oncol. 2012;33(1):42-50.

25. Vlad C, Kubelac P, Onisim A, et al. Expression of CDCP1 and ADAM12 in the ovarian cancer microenvironment. JBUON. 2016;21(4):973-978.

26. Bochis OV, Fetica B, Vlad C, Achimas-Cadariu P, Irimie A. The importance of ubiquitin E3 ligases, $\mathrm{SCF}$ and $\mathrm{APC} / \mathrm{C}$, in human cancers. Clujul Med. 2015;88(1):9-14.

27. Irimie AI, Braicu C, Pileczki V, et al. Knocking down of $\mathrm{p} 53$ triggers apoptosis and autophagy, concomitantly with inhibition of migration on SSC-4 oral squamous carcinoma cells. Mol Cell Biochem. 2016;419(1-2):75-82.

28. Frinc I, Muresan MS, Zaharie F, et al. Cancer stem-like cells: the dark knights of clinical hematology and oncology. J BUON. 2014;19(2):328-335.

29. Hu Q, Hong L, Nie M, et al. The effect of dehydroepiandrosterone supplementation on ovarian response is associated with androgen receptor in diminished ovarian reserve women. J Ovarian Res. 2017;10(1):32.

30. Irimie A, Achimas-Cadariu P, Burz C, Puscas E. Multiple primary malignancies--epidemiological analysis at a single tertiary institution. J Gastrointestin Liver Dis. 2010;19(1):69-73.

31. Kubelac MP, Fetica B, Vlad IC, Fulop A, Popa A, Achimas-Cadariu P. The role of inhibitor of DNA-binding 1 (ID-1) protein and angiogenesis in serous ovarian cancer. Anticancer Res. 2014;34(1):413-416.

32. Vlad C, Kubelac P, Onisim A, Irimie A, Achimas-Cadariu P. The role of CDCP1 (CUB domain-containing protein 1) and ADAM12 (a disintegrin and metalloproteinase 12) in ovarian cancer. $J$ BUON. 2015;20(3):673-679.

33. Gherman C, Braicu OL, Zanoaga O, et al. Caffeic acid phenethyl ester activates pro-apoptotic and epithelial-mesenchymal transition-related genes in ovarian cancer cells A2780 and A2780cis. Mol Cell Biochem. 2016;413(1-2):189-198.

34. Haukvik UK, Dieset I, Bjoro T, Holte H, Fossa SD. Treatment-related premature ovarian failure as a long-term complication after Hodgkin's lymphoma. Ann Oncol. 2006;17(9):1428-1433.

35. Zheng Y, Thomas A, Schmidt CM, Dann CT. Quantitative detection of human spermatogonia for optimization of spermatogonial stem cell culture. Hum Reprod. 2014;29(11):2497-2511.

36. van der Kaaij MA, Heutte N, van Echten-Arends J, et al. Sperm quality before treatment in patients with early stage Hodgkin's lymphoma enrolled in EORTC-GELA Lymphoma Group trials. Haematologica. 2009;94(12):1691-1697.

37. Anselmo AP, Cartoni C, Bellantuono P, Maurizi-Enrici R, Aboulkair N, Ermini M. Risk of infertility in patients with Hodgkin's disease treated with ABVD vs MOPP vs ABVD/MOPP. Haematologica. 1990;75(2):155-158

38. Bonfante V, Santoro A, Viviani S, Valagussa P, Bonadonna G. ABVD in the treatment of Hodgkin's disease. Semin Oncol. 1992;19(2 Suppl 5):38-44; discussion 44-45.

39. Hodgson DC, Pintilie M, Gitterman L, et al. Fertility among female Hodgkin lymphoma survivors attempting pregnancy following ABVD chemotherapy. Hematol Oncol. 2007;25(1):11-15. 
40. Brusamolino E, Baio A, Orlandi E, et al. Long-term events in adult patients with clinical stage IA-IIA nonbulky Hodgkin's lymphoma treated with four cycles of doxorubicin, bleomycin, vinblastine, and dacarbazine and adjuvant radiotherapy: a single-institution 15-year follow-up. Clin Cancer Res. 2006;12(21):6487-6493.

41. Behringer K, Breuer K, Reineke T, et al. Secondary amenorrhea after Hodgkin's lymphoma is influenced by age at treatment, stage of disease, chemotherapy regimen, and the use of oral contraceptives during therapy: a report from the German Hodgkin's Lymphoma Study Group. J Clin Oncol. 2005;23(30):7555-7564.

42. Decanter C, Morschhauser F, Pigny P, Lefebvre C, Gallo C, Dewailly D. Anti-Mullerian hormone follow-up in young women treated by chemotherapy for lymphoma: preliminary results. Reprod Biomed Online. 2010;20(2):280-285.

43. Yuksel A, Bildik G, Senbabaoglu F, et al. The magnitude of gonadotoxicity of chemotherapy drugs on ovarian follicles and granulosa cells varies depending upon the category of the drugs and the type of granulosa cells. Hum Reprod. 2015;30(12):2926-2935.

44. Lambertini M, Del Mastro L, Viglietti G, Ponde NF, Solinas C, de Azambuja E. Ovarian function suppression in pemenopausal women with early-stage breast cancer. Curr Treat Options Oncol. 2017;18(1):4.

45. Harel S, Ferme C, Poirot C. Management of fertility in patients treated for Hodgkin's lymphoma. Haematologica. 2011;96(11):1692-1699.

46. Leroy C, Rigot JM, Leroy M, et al. Immunosuppressive drugs and fertility. Orphanet J Rare Dis. 2015;10:136.

47. Loren AW. Fertility issues in patients with hematologic malignancies. Hematology Am Soc Hematol Educ Program. 2015;2015:138-145.

48. Ash P. The influence of radiation on fertility in man. Br J Radiol. 1980;53(628):271-278.

49. Nakayama K, Liu P, Detry M, et al. Receiving information on fertility- and menopause-related treatment effects among women who undergo hematopoietic stem cell transplantation: changes in perceived importance over time. Biol Blood Marrow Transplant. 2009; 15(11):1465-1474.

50. Swerdlow AJ, Cooke R, Bates A, et al. Risk of premature menopause after treatment for Hodgkin's lymphoma. J Natl Cancer Inst. 2014;106(9).

51. de Bruin ML, Huisbrink J, Hauptmann M, et al. Treatment-related risk factors for premature menopause following Hodgkin lymphoma. Blood. 2008;111(1):101-108.

52. Kapke JT, Epperla N, Shah N, et al. Effect of routine surveillance imaging on the outcomes of patients with classical Hodgkin lymphoma after autologous hematopoietic cell transplantation. Clin Lymphoma Myeloma Leuk. 2017;17(7):408-414.

53. Wilke C, Cao Q, Dusenbery KE, et al. Role of consolidative radiation therapy after autologous hematopoietic cell transplantation for the treatment of relapsed or refractory Hodgkin lymphoma. Int J Radiat Oncol Biol Phys. 2017;99(1):94-102.

54. Padberg S, Mick I, Frenzel C, Greil R, Hilberath J, Schaefer C. Transient congenital dilated cardiomyopathy after maternal R-CHOP chemotherapy during pregnancy. Reprod Toxicol. 2017;71:146-149.

55. Bragnes Y, Boshuizen R, de Vries A, Lexberg A, Ostensen M. Low level of Rituximab in human breast milk in a patient treated during lactation. Rheumatology. 2017;56(6):1047-1048.

56. Prince HM, Kim YH, Horwitz SM, et al. Brentuximab vedotin or physician's choice in CD30-positive cutaneous T-cell lymphoma (ALCANZA): an international, open-label, randomised, phase 3, multicentre trial. Lancet. 2017;390(10094):555-566.

57. Bair SM, Strelec L, Nagle SJ, et al. Outcomes of patients with relapsed/ refractory Hodgkin lymphoma progressing after autologous stem cell transplant in the current era of novel therapeutics: a retrospective analysis. Am J Hematol. 2017;92(9):879-884.

58. Gafencu GA, Selicean SE, Petrushev B, et al. Clinicopathological analysis of a case series of peripheral T-cell lymphomas, not otherwise specified, of lymphoepithelioid variant (Lennert's lymphoma). A Central European single-center study. Hum Pathol. 2016;53:192-194.
59. Dima D, Tomuleasa C, Irimie A, et al. Magnetic resonance imagingbased diagnosis of progressive multifocal leukoencephalopathy in a patient with non-Hodgkin lymphoma after therapy with cyclophosphamide, doxorubicin, vincristine, prednisone, and rituximab. Cancer. 2014;120(24):4005-4006.

60. Ansell SM. Brentuximab vedotin. Blood. 2014;124(22):3197-3200.

61. Scott LJ. Brentuximab vedotin: a review in CD30-positive Hodgkin lymphoma. Drugs. 2017;77(4):435-445.

62. Moskowitz $\mathrm{CH}$, Nademanee A, Masszi T, et al. Brentuximab vedotin as consolidation therapy after autologous stem-cell transplantation in patients with Hodgkin's lymphoma at risk of relapse or progression (AETHERA): a randomised, double-blind, placebo-controlled, phase 3 trial. Lancet. 2015;385(9980):1853-1862.

63. Kanematsu D, Shofuda T, Yamamoto A, et al. Isolation and cellular properties of mesenchymal cells derived from the decidua of human term placenta. Differentiation. 2011;82(2):77-88.

64. Onisim A, Achimas-Cadariu A, Vlad C, Kubelac P, Achimas-Cadariu P. Current insights into the association of Nestin with tumor angiogenesis. J BUON. 2015;20(3):699-706.

65. Flerlage JE, Metzger ML, Wu J, Panetta JC. Pharmacokinetics, immunogenicity, and safety of weekly dosing of brentuximab vedotin in pediatric patients with Hodgkin lymphoma. Cancer Chemother Pharmacol. 2016;78(6):1217-1223.

66. Ansell SM. Nivolumab in the treatment of Hodgkin lymphoma. Clin Cancer Res. 2017;23(7):1623-1626.

67. Lesokhin AM, Ansell SM, Armand P, et al. Nivolumab in patients with relapsed or refractory hematologic malignancy: preliminary results of a phase Ib study. J Clin Oncol. 2016;34(23):2698-2704.

68. Merryman RW, Kim HT, Zinzani PL, et al. Safety and efficacy of allogeneic hematopoietic stem cell transplant after PD-1 blockade in relapsed/refractory lymphoma. Blood. 2017;129(10):1380-1388.

69. Lin RJ, Diefenbach CS. Checkpoint inhibition in Hodgkin lymphoma: saving the best for last? Oncology (Williston Park). 2016;30(10):914-920.

70. Pembrolizumab approved for Hodgkin lymphoma. Cancer Discov. 2017;7(5):OF1.

71. Chen R, Zinzani PL, Fanale MA, et al. Phase II study of the efficacy and safety of pembrolizumab for relapsed/refractory classic Hodgkin lymphoma. J Clin Oncol. 2017;35(19):2125-2132.

72. Armand P, Shipp MA, Ribrag V, et al. Programmed death-1 blockade with pembrolizumab in patients with classical Hodgkin lymphoma after brentuximab vedotin failure. J Clin Oncol. 2016;34(31):3733-3739.

73. Ellinger I, Fuchs R. HFcRn-mediated transplacental immunoglobulin $\mathrm{G}$ transport: protection of and threat to the human fetus and newborn. Wien Med Wochenschr. 2012;162(9-10):207-213.

74. Warrington JP, Drummond HA, Granger JP, Ryan MJ. Placental ischemia-induced increases in brain water content and cerebrovascular permeability: role of TNF- $\alpha$. Am J Physiol Regul Integr Comp Physiol. 2015;309(11):R1425-1431.

75. Pfreundschuh M, German High-Grade Non-Hodgkin Lymphoma Study Group (DSHNHL). High-dose chemotherapy and autologous stem-cell transplantation for DLBCL in the rituximab era. Lancet Oncol. 2017;18(8):989-991.

76. Della Pepa R, Picardi M, Giordano C, et al. Rituximab in a riskadapted treatment strategy gives excellent therapeutic results in nodular lymphocyte-predominant Hodgkin lymphoma. Br J Haematol. 2017. [Epub ahead of print].

77. Tomuleasa C, Braicu C, Irimie A, Craciun L, Berindan-Neagoe I. Nanopharmacology in translational hematology and oncology. Int $J$ Nanomedicine. 2014;9:3465-3479.

78. Chakravarty EF, Murray ER, Kelman A, Farmer P. Pregnancy outcomes after maternal exposure to rituximab. Blood. 2011;117(5):1499-1506.

79. Thurn L, Lindqvist PG, Jakobsson M, et al. Abstracts of the 26th World Congress on Ultrasound in Obstetrics and Gynecology, Rome, Italy, 24-28 September 2016. Ultrasound Obstet Gynecol. 2016;48(Suppl 1):140. 
80. Broer SL, Broekmans FJ, Laven JS, Fauser BC. Anti-Mullerian hormone: ovarian reserve testing and its potential clinical implications. Hum Reprod Update. 2014;20(5):688-701.

81. Gultekin M, Dursun P, Vranes B, et al. Gynecologic oncology training systems in Europe: a report from the European network of young gynaecological oncologists. Int J Gynecol Cancer. 2011;21(8):1500-1506.

82. Ordeanu C, Pop DC, Badea R, et al. Local experience in cervical cancer imaging: Comparison in tumour assessment between TRUS and MRI. Rep Pract Oncol Radiother. 2015;20(3):223-230.

83. Mahany EB, Randolph JF Jr. Biochemical and imaging diagnostics in endocrinology: predictors of fertility. Endocrinol Metab Clinics North Am. 2017;46(3):679-689.

84. Iraha Y, Okada M, Iraha R, et al. CT and MR imaging of gynecologic emergencies. Radiographics. 2017;37(5):1569-1586.

85. Lambertini M, Cinquini M, Moschetti I, et al. Temporary ovarian suppression during chemotherapy to preserve ovarian function and fertility in breast cancer patients: a GRADE approach for evidence evaluation and recommendations by the Italian Association of Medical Oncology. Eur J Cancer. 2017;71:25-33

86. Bedaiwy MA, Allaire C, Alfaraj S. Long-term medical management of endometriosis with dienogest and with a gonadotropin-releasing hormone agonist and add-back hormone therapy. Fertil Steril. 2017;107(3):537-548.

87. Huser M, Smardova L, Janku P, et al. Fertility status of Hodgkin lymphoma patients treated with chemotherapy and adjuvant gonadotropin-releasing hormone analogues. J Assist Reprod Genet. 2015;32(8):1187-1193.

88. Falorio S, Angrilli F, Fioritoni G. Gonadotropin-releasing hormone analog treatment for the prevention of treatment-related ovarian failure and infertility in women of reproductive age with Hodgkin lymphoma. Leuk Lymphoma. 2008;49(6):1087-1093.

89. Blumenfeld Z, Avivi I, Eckman A, Epelbaum R, Rowe JM, Dann EJ. Gonadotropin-releasing hormone agonist decreases chemotherapyinduced gonadotoxicity and premature ovarian failure in young female patients with Hodgkin lymphoma. Fertil Steril. 2008;89(1):166-173.

90. Nitzschke M, Raddatz J, Bohlmann MK, Stute P, Strowitzki T, von Wolff M. GnRH analogs do not protect ovaries from chemotherapyinduced ultrastructural injury in Hodgkin's lymphoma patients. Arch Gynecol Obstet. 2010;282(1):83-88.

91. Behringer K, Wildt L, Mueller H, et al. No protection of the ovarian follicle pool with the use of $\mathrm{GnRH}$-analogues or oral contraceptives in young women treated with escalated BEACOPP for advanced-stage Hodgkin lymphoma. Final results of a phase II trial from the German Hodgkin Study Group. Ann Oncol. 2010;21(10):2052-2060.

92. Sonigo C, Seroka A, Cedrin-Durnerin I, Sermondade N, Sifer C, Grynberg M. History of ABVD alters the number of oocytes vitrified after in vitro maturation in fertility preservation candidates. Future Oncol. 2016;12(14):1713-1719.

93. Alvarez RM, Ramanathan P. Fertility preservation in female oncology patients: the influence of the type of cancer on ovarian stimulation response. Hum Reprod. 2016; pii: dew158. [Epub ahead of print].

94. Dahhan T, Balkenende EME, Beerendonk CCM, et al. Stimulation of the ovaries in women with breast cancer undergoing fertility preservation: Alternative versus standard stimulation protocols; the study protocol of the STIM-trial. Contemp Clin Trials. 2017;61:96-100.

95. Pereira N, Hubschmann AG, Lekovich JP, Schattman GL, Rosenwaks Z. Ex vivo retrieval and cryopreservation of oocytes from oophorectomized specimens for fertility preservation in a BRCA1 mutation carrier with ovarian cancer. Fertil Steril. 2017;108(2):357-360.
96. Rienzi L, Cobo A, Paffoni A, et al. Consistent and predictable delivery rates after oocyte vitrification: an observational longitudinal cohort multicentric study. Hum Reprod. 2012;27(6):1606-1612.

97. Donnez J, Martinez-Madrid B, Jadoul P, Van Langendonckt A, Demylle D, Dolmans MM. Ovarian tissue cryopreservation and transplantation: a review. Hum Reprod Update. 2006;12(5):519-535.

98. Binesh F, Akhavan A, Behniafard N, Atefi A. Clinicopathologic and survival characteristics of childhood and adolescent non Hodgkin's lymphoma in Yazd, Iran. Asian Pac J Cancer Prev. 2014;15(4): $1585-1588$.

99. Anselmo AP, Cavalieri E, Aragona C, Sbracia M, Funaro D, Maurizi Enrici R. Successful pregnancies following an egg donation program in women with previously treated Hodgkin's disease. Haematologica. 2001;86(6):624-628

100. Evenson DP, Jost LK, Marshall D, et al. Utility of the sperm chromatin structure assay as a diagnostic and prognostic tool in the human fertility clinic. Hum Reprod. 1999;14(4):1039-49.

101. Sergerie M, Laforest G, Bujan L, Bissonnette F, Bleau G. Sperm DNA fragmentation: threshold value in male fertility. Hum Reprod. 2005;20(12):3446-3451.

102. Morris ID, Ilott S, Dixon L, Brison DR. The spectrum of DNA damage in human sperm assessed by single cell gel electrophoresis (Comet assay) and its relationship to fertilization and embryo development. Hum Reprod. 2002;17(4):990-998.

103. Zubkova EV, Wade M, Robaire B. Changes in spermatozoal chromatin packaging and susceptibility to oxidative challenge during aging. Fertil Steril. 2005;84(Suppl 2):1191-1198.

104. Redig AJ, Brannigan R, Stryker SJ, Woodruff TK, Jeruss JS. Incorporating fertility preservation into the care of young oncology patients Cancer. 2011;117(1):4-10.

105. Howell SJ, Shalet SM. Spermatogenesis after cancer treatment: damage and recovery. J Natl Cancer Inst Monogr. 2005;(34):12-17.

106. Sieniawski M, Reineke T, Josting A, et al. Assessment of male fertility in patients with Hodgkin's lymphoma treated in the German Hodgkin Study Group (GHSG) clinical trials. Ann Oncol. 2008;19(10):1795-1801.

107. Loren AW, Mangu PB, Beck LN, et al. Fertility preservation for patients with cancer: American Society of Clinical Oncology clinical practice guideline update. J Clin Oncol. 2013;31(19):2500-2510.

108. Sucala M, Cuijpers P, Muench F, et al. Anxiety: there is an app for that. A systematic review of anxiety apps. Depress Anxiety. 2017;34(6):518-525.

109. Tomuleasa C, Zaharie F, Muresan MS, et al. How to diagnose and treat a cancer of unknown primary site. $J$ Gastrointest Liver Dis. 2017;26(1):69-79.

110. Braicu C, Chiorean R, Irimie A, et al. Novel insight into triple-negative breast cancers, the emerging role of angiogenesis, and antiangiogenic therapy. Expert Rev Mol Med. 2016;18:e18.

111. Frinc I, Ilies P, Zaharie F, et al. Transthoracic ultrasonography for the immunocompromised patient. A pilot project that introduces transthoracic ultrasonography for the follow-up of hematological patients in Romania. Rom J Intern Med. 2017;55(2):103-116.

112. Micu R, Petrut B, Zlatescu-Marton C, Traila A, Harsa R, AchimasCadariu P. Current strategies and future perspectives in fertility preservation for cancer patients. J BUON. 2017;22(4):844-852.

113. 113. Truta Z, Garlovanu M, Lerintiu S, Micu R. A new method for human semen glucose concentration evaluation. Rom Biotech Lett 2010;15:5764-5772. 


\section{Publish your work in this journal}

Cancer Management and Research is an international, peer-reviewed open access journal focusing on cancer research and the optimal use of preventative and integrated treatment interventions to achieve improved outcomes, enhanced survival and quality of life for the cancer patient. The manuscript management system is completely online and includes a very quick and fair peer-review system, which is all easy to use. Visit http://www.dovepress.com/testimonials.php to read real quotes from published authors. 